\title{
Kernos
}

Revue internationale et pluridisciplinaire de religion grecque antique

$3 \mid 1990$

Varia

\section{Autorité mantique et autorité politique : Tirésias et Oedipe}

Antonis Paparizos

\section{(apenEdition \\ Journals}

\section{Édition électronique}

URL : http://journals.openedition.org/kernos/1000

DOI : 10.4000/kernos. 1000

ISSN : 2034-7871

\section{Éditeur}

Centre international d'étude de la religion grecque antique

Édition imprimée

Date de publication : 1 janvier 1990

ISSN : 0776-3824

\section{Référence électronique}

Antonis Paparizos, «Autorité mantique et autorité politique : Tirésias et Oedipe », Kernos [En ligne], 3 | 1990, mis en ligne le 19 avril 2011, consulté le 21 avril 2019. URL : http://journals.openedition.org/ kernos/1000 ; DOI : 10.4000/kernos.1000 


\section{AUTORITÉ MANTIQUE ET AUTORI'TÉ POLITIQUE : TIRÉSIAS ET OEDIPE}

\section{La Question}

Dans la tragédie de Sophocle Philoctète, nous apprenons que Hélénos, fils de Priam et devin reconnu, pris dans un piège par Ulysse, se voit obligé de prédire aux Achéens ce qu'ils doivent faire pour conquérir Troie. De même, dans l'Odyssée, Ménélas et ses compagnons, immobilisés dans l'île de Pharos, parviennent, en suivant les conseils d'Idothée, à forcer le vieux et immortel Protée à avouer de quelle manière ils pourraient apaiser la colère des dieux et regagner leur patrie. Ulysse, d'autre part, lors de sa descente aux Enfers, obtient de Tirésias la connaissance de son destin. Par contre, dans une autre tragédie de Sophocle, Edipe-roi, nous avons un paradigme tout à fait différent. CEdipe et Tirésias s'affrontent, à titre égal, sur plusieurs niveaux d'autorité, et à partir d'intérêts contradictoires.

La question qui se pose est donc la suivante : si les instances divinatoires, individus ou institutions, sont obligées d'exercer leur art mantique au profit de ceux qui se servent de la force, du pouvoir ou encore de la violence physique, y a-t-il vraiment une forme d'autorité mantique quelconque ? Et si la réponse est positive, quels peuvent en être les fondements et le contenu?

Certes, nous ne pouvons pas nous occuper, ici, du problème dans son ensemble. Plus modestement, nous nous proposons d'étudier ces mêmes questions en partant d'un seul paradigme, celui de l'affrontement entre Tirésias et Edipe. Par ailleurs, nous sommes obligé d'essayer de répondre à une question qui de toute manière se pose implicitement : pourquoi l'autorité mantique ne s'est-elle jamais investie ou transformée en autorité ou en pouvoir politique, ce qui a très souvent été le cas de l'autorité religieuse?

Notre exposé comporte deux parties. Dans la première, nous tenterons de traiter des questions méthodologiques et théoriques, tandis que la seconde étudiera la question concrète, afin d'en tirer des conclusions de portée générale. 


\section{Partie théorique}

\section{De l'autorité}

$\mathrm{Si}$ par politique on entend la forme d'«unité» fondamentale en conséquence de laquelle les hommes émergent en tant que société, «unité» matériellement présente dans les rapports de force qui se développent entre les êtres humains, les groupes et les classes sociales, l'autorité ne peut constituer que des formes de cristallisation et/ou de matérialisation de cette "unité»" ${ }^{1}$, aussi bien chez les êtres que dans les institutions.

Cela signifie également que le politique et, par conséquent, l'autorité, en tant que fondements de toute forme d'organisation sociale, peuvent se cristalliser et se réaliser, d'une part, sous forme de violence ouverte $^{2}$, physique et/ou économique, dans les sociétés politiques à coercition et, d'autre part, sous forme d'équilibre continu et immanent aux forces humaines et sociales, dans les sociétés sans coercition ${ }^{3}$.

«[...] une certaine autorité, écrit Engels, et une certaine soumission sont des choses qui, indépendamment de toute forme d'organisation sociale, s'imposent à nous par les conditions matérielles (nous dirions

1 On peut appréhender le politique en partant du pouvoir, de l'État ou, en général, du «phénomène politique», pour ne citer que les acceptions principales. Nous essayons de définir le politique comme une forme fondamentale d'«unité» sociale : principe d'organisation de la vie des hommes. L'homme émerge et vit toujours en société. Toute forme d'organisation de la vie humaine ne peut, étant donné les énormes contradictions et la lutte interne des groupes et des classes sociales, qu'être une forme «d'unité forcée» et, ainsi, une forme d'unité politique. Cf. notre thèse d'État: Contribution à une appréhension du rapport entre politique et religion, Paris, $1977,547 \mathrm{p}$. Certes, il faut bien distinguer le politique de la politique. Toute société est politique mais ce n'est que dans l'antiquité hellénique que la politique émerge. Voir à cet égard C. CASTORIADIs, Pouvoir, politique, autonomie, in $R M M$ (1988), p. 92 sq.

2 Sur les formes de la violence, «les modes de domination», cf. P. BourdiEU, Le sens pratique, Paris, 1980, surtout p. 216 sq.

3 Sociétés «étatiques» et sociétés sans État. Diverses sont les questions qui se posent à cet égard. À titre indicatif, voir P. CLASTRES, Société contre l'État, Paris, 1974; J.-W. LAPIERRE, Essai sur le fondement du pouvoir politique, Aix-enProvence, 1968. Paradigme de société sans chef et sans prêtre, cf. M. GoDELIER, Horizon, trajets marxistes en anthropologie, Paris, 1977, t. II, chap. V. 
également symboliques ${ }^{4}$ ), sous lesquelles nous produisons et nous distribuons les produits ${ }^{5}$.

L'autorité, en tant que rapport de force, est présente dans les rapports de production et de distribution ou, autrement dit, dans le processus économique. Et si cela est vrai, il va de soi qu'elle est présente dans toute forme de rapport entre les hommes, les groupes et les classes sociales, même dans les relations strictement personnelles.

Tout se passe comme si l'autorité était la «chance» 6 ou, peut-être, la "possibilité» pour les instances sociales, individus et institutions, de faire accepter la parole ${ }^{7}$ et leur volonté, et/ou de mobiliser la force matérielle et symbolique des groupes et des classes sociales, en leur offrant des biens et des services matériels et symboliques capables de satisfaire leurs intérêts et leurs besoins. Ainsi, l'autorité peut également se réaliser et s'acquérir dans et par la "chance» de certaines instances sociales de produire, surtout en périodes de transformation, de nouvelles formes de biens, dont le besoin, diffus jusqu'alors dans le corps social, n'a pas été cristallisé, structuré et, par conséquent, ressenti. C'est le travail des grands prophètes, révolutionnaires et réformateurs de l'histoire.

Si nous définissons l'autorité comme «chance» et/ou comme «possibilité», c'est pour deux raisons. D'abord parce qu'elle ne constitue pas un élément donné, et comme tel constant, de la conscience ou de la

4 Au sujet des «biens symboliques», cf. P. Bourdieu, Le marché des biens symboliques, in L'Année sociologique, 22 (1971), p. 49-126.

5 Cette citation constitue la proposition principale d'un article d'ENGELS paru pour la premiere fois en italien en 1874 sous le titre Dell' autorita, in Almanacco Repubblicano et, en 1959, en allemand. Nous utilisons ici la traduction grecque, in Deucalion, 22 (1978), p. 244-247.

6 La «chance» constitue un schéma fondamental dans la manière dont $M$. WEBER appréhende la puissance, la domination et les formes de domination : Economie et société, Paris, 1971. Nous utilisons cette notion afin de compléter notre définition de l'autorité en tant que «rapport de force». Pour qu'un rapport de force s'établisse, il faut avoir une "chance" ou, autrement dit, une "possibilité». Les conditions historiques ne peuvent fonctionner que comme *conditions de possibilitém.

7 La parole est la première forme de force dont on se sert pour s'imposer. K. MARX, qui n'a pas fait une théorie de l'autorité, en a eu conscience. Il parle de la «violence sans parole» (de l'exécutif), et de la «violence des paroles» (du législatif); 18 Brumaire de Louis Bonaparte, Thènes, 1975, p. 152. P. BouRdIEU nous parlera de la «violence symbolique, par rapport à la violence physique, dans son ouvrage, écrit avec J.-C. PASseron, La reproduction, Paris, 1970, p. $13 \mathrm{sq}$. 
psychologie ${ }^{8}$ de l'homme, indépendamment des conditions et des structures économiques et politiques d'une société historiquement située. Et, d'autre part, parce qu'elle peut s'instituer et se matérialiser dans et par les trois types fondamentaux de rapports sociaux. Rapports politiques, en tant que rapports de force, de combat et de mobilisation. Rapports économiques, en tant que rapports de production et de distribution des biens matériels. Et, enfin, rapports symboliques qui investissent les deux autres espèces de rapports d'un contenu idéologique et significatif. Il faut ajouter également que l'autorité prend la forme du pouvoir et de la domination lorsque les rapports d'unité politique deviennent, au sein d'une société, ouvertement violents9.

Si l'autorité est présente comme "chance" dans toute forme de rapports sociaux, elle s'exerce et elle obtient ses formes et son contenu concret au sein d'un champ de production des biens et, en même temps, de force et de domination. C'est la raison pour laquelle nous devons faire une esquisse, même très schématique, de la structure du champ de production et d'autorité mantiques, surtout en rapport avec le champ politique.

\section{Champ de production et d'autorité mantiques et champ politique}

Par champ nous entendons un «lieu» d'action qui se compose de trois structures de rapports sociaux. Structure des rapports économiques de production et de distribution des biens, structure des rapports politiques de force, de lutte et/ou de domination, et structure des rapports symboliques de force et de lutte idéologique ${ }^{10}$. Ces trois structures peuvent être

8 Il est certain que l'autorité aussi bien que le pouvoir trouvent des fondements dans la psychologie de l'homme. On ne saurait nier cette réalité et on ne saurait faire de la sociologie sans tenir compte de la psychologie humaine, sociale et politique. Le «désir» est présent dans toute forme d'activité humaine. Mais on ne saurait pas non plus admettre que l'homme se caractérise par des tendances constantes et inchangeables à se soumettre et/ou à dominer, tendances qui pourraient être conçues comme des «constantes anthropologiques».

9 Même dans les sociétés où nous n'avons pas de pouvoir, de domination et/ou d'hiérarchie, nous avons de l'autorité. Le plan implicite et immanent dans et par lequel s'organisent ces sociétés constitue sa matérialité. C'est également celle-ci qui prend la forme du pouvoir et de la domination lorsque les rapports humains deviennent violents.

10 Diverses sont les questions théoriques qui concernent la conception d'un champ de production et de domination. Il est impossible de les traiter ici. Nous en avons un très bon exemple dans l'article de P. BouRdieu, Genèse et structure du champ religieux, in Revue française de sociologie (1971), p. 295-334, qui nous a donné l'idée d'un champ de production mantique. 
calquées l'une sur l'autre, mais elles peuvent également se trouver en rapport d'autonomie relative. Cela dépend d'une part de l'activité des hommes et, d'autre part, de la complexité qui tient aux conditions historiques de l'évolution de chaque société concernée ${ }^{11}$.

Par conséquent, le champ mantique de l'antiquité hellénique, si l'on étudie l'histoire de sa formation ${ }^{12}$, s'institue dans et par le marché de biens mantiques, la structure des rapports d'autorité qui en découle et la longue tradition cultuelle, avec toutes les significations et les explications sur la vie des hommes qu'elle comporte : conception ou conceptions de l'homme, de la société et du monde. À partir de là, les questions qui se posent concernent les producteurs des biens mantiques, individus et institutions, les espèces et les formes systématisées du travail de divination, les différents types de produits mantiques et, enfin, les formes de lutte, s'il y en a, entre les producteurs pour l'acquisition de l'autorité.

Comme producteurs de biens mantiques, nous pouvons considérer les "diseurs d'oracles" et les "guérisseurs», les «bakis» et les "Sibylles", les "faiseurs de miracles» et encore tout sanctuaire ou tout autre institution qui pratique l'art de la divination. Les biens mantiques, par conséquent, sont en principe des «biens de vérité». Autrement dit, des conseils et des solutions, et en général des réponses qui concernent tout aspect matériel et symbolique de la vie quotidienne, aussi bien de l'oikos que de la polis. Il s'agit des «dires» et des "prédires», des «homologies» portant surtout sur des problèmes occasionnels, qui s'imposent et exigent une solution immédiate.

D'autre part, l'art mantique est un travail d'inspiration personnelle qui consiste à mobiliser les intentions, les pensées et les actes des hommes et/ou des groupes dans une direction prévue. Il serait très difficile de remonter aux origines de sa première formation, mais nous pouvons constater que, dès son émergence, nous avons plusieurs types d'opération divinatoire. Les producteurs des «biens de vérité", devins ou sanctuaires, développent chacun leur propre tradition, tout en se tenant

11 Il est impossible de débattre ici la question des rapports entre les structures économiques, politiques et symboliques. Pour ce débat : N. PoulanTZas, Pouvoir politique et classes sociales, Paris, 1968; K. POLANYI, La grande transformation, Paris, 1983; M. GoDELIER, Horizon... op. cit. , I, chap. I.

12 Les outils dont nous nous sommes servi pour appréhender le marché et le champ mantiques dans l'antiquité grecque sont, entre autres : L. GERNET et A. BoulANGER, Le génie grec dans la religion, Paris, 1970 (1932); A. BouchtLeClerCQ, Histoire de la divination dans l'antiquité, Paris, 1879-1882 (les trois premiers volumes); J.-P. VERNANT (éd.), Divination et rationalité, Paris, 1974. 
dans les grands courants de la tradition nationale et à partir de conditions qui leur sont spécifiques, c'est-à-dire mythe d'origine ${ }^{13}$, histoire de la région, histoire du sanctuaire, histoire personnelle du devin, guerres ou autres.

En effet, en essayant de satisfaire les besoins et les demandes qui surgissent sur le marché des biens mantiques de l'antiquité hellénique (un marché national a des aspects régionaux), les devins et les sanctuaires sont libres de systématiser et d'organiser leur travail d'après leur propre capacité d'inventer. Nous avons ainsi, entre autres, la pyromancie, la cléromancie, la nécromancie, l'ornithomancie que les devins pratiquent, plus ou moins, chacun à sa manière, et toute autre forme de divination élaborée dans les grands sanctuaires. Mais il est deux qualités qui leur font défaut par rapport aux biens religieux. Ils ne peuvent satisfaire ni les besoins durables de contenu existentiel qui concernent les questions de la vie, du destin et de la mort que s'il s'agit de destins individuels, ni les besoins des hommes à justifier leur classe sociale et leur situation économique ${ }^{14}$. De plus, et cela constitue leur particularité et, en même temps, leur «faiblesse», ils sont toujours soumis à l'épreuve de la vérité.

Cette évolution et cette organisation du travail divinatoire a eu deux conséquences très importantes. D'abord, les producteurs des «biens de vérité» ne se sont jamais organisés en un corps de professionnels hiérarchiquement structuré. Ensuite, de l'activité mantique n'a jamais émergé, dans le monde grec ancien, une institution capable, sinon de dominer, du moins de définir ou de contrôler les règles générales de la production mantique au niveau national. Les plus grandes institutions étaient les grands sanctuaires régionaux.

De ces deux conséquences découle une troisième. Du moment où il est impossible d'avoir un corps de professionnels et une institution qui domineraient la production mantique dans son ensemble, il est également impossible d'avoir une lutte quelconque pour la conquête du capital d'autorité, soumise à des règles et, par conséquent, un champ d'autorité mantique structuré par une logique propre et interne de

13 Le mythe d'origine doit avoir une très grande importance dans l'acquisition de l'autorité mantique. Il s'agit de la première institution d'un devin ou d'un sanctuaire, c'est-à-dire le capital d'autorité de départ, qui serait difficilement acquis sans un mythe quelconque. Et si l'on tient compte des genos de devins, la parenté semble aussi instituer un capital d'autorité de départ.

14 Sur la question, cf. M. WEBER, Économie et société, Paris, 1971, surtout p. 450452; ainsi que "savoir sacré, prédication, cure d'âmes, p. 481-490; "ordres, classes et religions", p. 491-534; P. BouRdiEU, Genèse... op. cit., p. 300-318. 
production et de domination. Il devient donc clair que si tout champ politique est défini par une logique de contradictions et de lutte pour l'acquisition de la force et du pouvoir, le champ mantique, en raison des propriétés spécifiques de l'activité divinatoire, n'a jamais connu une structure analogue à celle des champs politiques. Il a toujours été ouvert à tous ceux qui, individus ou institutions, pouvaient exercer l'art mantique et se justifier dans cette activité.

Par conséquent, tout se passe comme si le travail mantique était libre et indépendant au sein d'un champ de production et de distribution des biens mantiques, national et ouvert, non hiérarchisé et sans structure interne dominante d'autorité et de production. L'autorité mantique ne dépend ni de la structure du marché des biens de divination, ni de la structure d'un corps de professionnels qui possède ses règles de lutte. L'autorité mantique dépend de la "chance» des producteurs de «biens de divination" de répondre efficacement aux demandes et aux besoins des différentes instances sociales quelles qu'elles soient, individus ou institutions, familles ou cités entières.

Nous pouvons donc comprendre pourquoi elle n'a jamais pu s'investir ou se transformer en autorité politique et pourquoi les détenteurs de l'art divinatoire n'ont jamais pu se transformer en hommes d'action politique. Cela aurait pu se faire si le marché ou le champ d'autorité mantique avait coïncidé avec un champ d'autorité ou de lutte politique, ce qui a été très souvent le cas pour l'autorité religieuse.

\section{L'affrontement entre GEdipe et Tirésias}

L'histoire est très connue, ce qui nous dispense de nous y référer en détail et à tout moment. Edipe, après avoir appris que, pour sauver Thèbes il fallait trouver et mettre à mort ou exiler le meurtrier de l'ancien roi Laios, a fait appeler Tirésias en espérant obtenir une indication quelconque sur l'identité du coupable. La rencontre et l'affrontement commencent lorsque le devin se présente devant la cité et devant le roi. La lutte comprend trois étapes, et la première, deux phases.

\section{La lutte au nom de la cité (300-344)}

Lorsque Tirésias arrive, Edipe, en lui adressant la parole et en lui accordant les titres que l'on attribuait habituellement au roi, $\pi \rho 0 \sigma \tau \alpha \dot{\tau} \tau \eta$, $\sigma \omega \tau \hat{p} \rho \alpha$ et $\alpha$ $v \alpha \xi$ (303-304), le place à son propre niveau d'autorité. Aussitôt après, et dans le même discours, il concède à Tirésias les mêmes intérêts à prendre la parole que les siens propres, à savoir se sauver lui- 


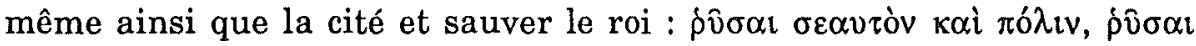
$\delta^{\prime} \dot{\varepsilon} \mu \dot{\varepsilon}(312)$.

Tirésias, par contre, ne semble pas du tout se laisser influencer par les honneurs qu'on lui fait et, à la grande surprise d'Edipe, refuse de

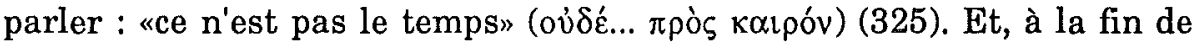
cette première phase (300-329) de la première étape, CEdipe en vient à le supplier au nom de la cité (327), ce qui ne le met pas, forcément, en état d'infériorité par rapport à Tirésias. L'égalité symbolique de l'autorité des deux hommes reste intacte, mais au niveau des intérêts, elle est déjà renversée. L'intérêt de la cité ne semble pas pouvoir influencer Tirésias.

Durant la seconde phase de la première étape (330-344), l'ambiance de départ change complètement. L'affrontement commence. CEdipe attaque Tirésias en l'accusant de trahison envers les citoyens et en lui rappelant à deux reprises $(331,340)$ ses obligations envers la cité. Tirésias refuse de parler parce qu'il ne veut pas provoquer de tristesse (332) : «les choses se montreront d'elles-mêmes» (341). Et il fait savoir que la colère d'Edipe lui est complètement indifférente (344).

À propos de cette première étape, les points les plus importants sont les suivants. Nous sommes devant deux formes d'autorité complètement différentes. L'autorité d'Edipe possède trois fondements : le «charisme» personnel d'un homme qui a su se servir de sa capacité de penser et d'inventer, le fait d'avoir sauvé Thèbes de la Sphinx et le pouvoir politique d'un chef légitime. Ce dernier fondement se réalise dans et par le champ d'autorité que constitue le palais avec les membres de la famille royale, et dans le champ politique de la cité qui englobe tous les citoyens. L'autorité de Tirésias, par contre, dérive de sa capacité personnelle de satisfaire, comme serviteur d'Apollon, les besoins en «biens de vérité» de tous ceux qui s'adressent à lui.

Ces deux formes d'autorité, cependant, s'instituent par les mêmes symboles et possèdent la même force durant la lutte. Il est certain qu'EEdipe attribue à Tirésias presque les mêmes titres d'autorité que lui ont accordés les citoyens de Thèbes lorsqu'ils sont venus lui demander de les sauver une seconde fois. Et il est également certain que les deux hommes, durant cette première étape, s'affrontent à titre égal. Comment pouvons-nous expliquer ce phénomène? Le statut politique de Tirésias est double. D'une part, il est un devin à l'autorité reconnue qui, vu les circonstances difficiles, se place au niveau de l'autorité royale. CEdipe ne fait que reconnaître cette réalité. D'autre part, en tant que Thébain, il jouit de tous les droits d'un citoyen. 
L'autorité de Tirésias, sans se transformer en autorité politique, se déploie de la même manière. D'autre part, en tant que devin, il garde son indépendance et sa force de protégé d'Apollon, ce qui lui permet de rester indifférent à l'intérêt de la cité et à la colère d'EEdipe. En tout cas, il connaît la vérité et sait que "les choses vont suivre leur évolution". Nous constatons, par conséquent, que l'autorité de Tirésias trouve dans le champ politique de Thèbes un second fondement, sans pour autant se transformer en autorité politique. Edipe ne peut se servir, pour le persuader, que des raisons ou des moyens légitimes. La force et la violence, dont s'étaient servis Ulysse et Ménélas contre Hélénos et Protée, sont complètement exclues. En tout cas, les formes d'autorité et de pouvoir qui s'exercent à Athènes et dans d'autres cités durant cette période sont démocratiques, et les tragédies de Sophocle semblent refléter positivement ce processus de démocratisation.

En partant de cette explication, nous pouvons également comprendre pourquoi Edipe se tient, tout au long de la première étape, au niveau de l'intérêt de la cité, en parlant toujours au nom de la cité, et Tirésias au niveau de l'intérêt personnel, en parlant du kairos et du "déroulement propre des choses", éléments qui constituent les principes de son art divinatoire. Edipe, homme charismatique, sauveur de Thèbes et roi, mobilise toute son autorité légitime pour sauver sa cité encore une fois. Il ne sait pas encore de quelle autre manière il est concerné. Tirésias, également charismatique, mobilise toute son autorité pour éviter, précisément, de dire la vérité.

\section{Le combat personnel (345-377)}

Les deux hommes se mettent mutuellement en cause et Tirésias commence à révéler ce qu'il sait du miasme, faisant ainsi de la vérité le vrai terrain de l'affrontement.

Edipe accuse Tirésias d'avoir lui-même participé, d'une manière ou d'une autre, au crime (345-349), mettant ainsi en cause la pureté du devin et, par conséquent, sa qualité de «diseur de vérité» (349). Cela fait exploser Tirésias qui commence à prononcer son oracle en révélant que le coupable est Edipe lui-même, ce qui met en cause l'identité politique et le charisme de ce dernier (353). CEdipe, frappé personnellement, ne parle plus de l'intérêt de la cité. Il révèle son pouvoir de roi et cherche à effrayer Tirésias en évoquant trois fois $(355,363,368)$, lors d'un dialogue très serré (354-377), sa capacité de le punir. Tirésias, de son côté, poursuit l'attaque en répétant ce qu'il vient de dire (362), dénonçant les relations affreuses d'CEdipe avec ses bien-aimés (367), et soulignant 
qu'il n'a rien à craindre parce qu'il se nourrit de la force de la vérité,

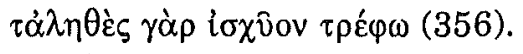

Cette seconde étape arrive à son terme avec une attaque d'Edipe et une contre-attaque de Tirésias. Le premier tente de ternir encore une fois la lumière que représente le devin en l'accusant de se nourrir de nuit (374). Tirésias, pour répondre, change le niveau de la lutte et fait comme s'il n'était pas lui-même son opposant, mais Apollon. "Ce n'est pas par moi que tu vas tomber, mais par Apollon" (377).

Les points sur lesquels nous devons mettre l'accent sont les suivants. Le combat surgit lorsque l'intérêt personnel de Tirésias, son identité de devin, c'est-à-dire la pureté et la lumière qui constituent l'essence d'un serviteur de la vérité, sont en cause. Dès ce moment, Tirésias est obligé de prouver son identité de serviteur d'Apollon. Son autorité en dépend. Pour ce faire, il n'y a qu'un seul moyen : annoncer la vérité, démontrer une fois encore sa capacité et son art.

Tirésias contre-attaque en mettant en cause l'identité d'Edipe. Tout se passe comme si la lutte se développait à deux niveaux qui se recouvrent : la vérité et l'autorité. Plus Tirésias avance dans l'énoncé de la vérité, plus son autorité se renforce. Chez lui, autorité et vérité semblent constituer un seul niveau : vérité signifie autorité et autorité signifie vérité. Par contre, plus Edipe exerce son pouvoir, plus il s'affaiblit et plus il doit lutter au niveau de la vérité. À la fin de cette étape, Edipe est dépouillé, vis-à-vis de Tirésias, de presque tout son pouvoir politique. Il ne lui restera qu'un seul fondement d'autorité : son charisme personnel et son savoir.

Un point très intéressant de cette étape se trouve dans l'aveu de Tirésias selon lequel ce n'est pas lui qui va perdre CEdipe, mais Apollon, comme si la véritable opposition ne se jouait pas entre CEdipe et lui, mais entre CEdipe et Apollon. Tirésias n'est que la voix d'Apollon, la «voix aveugle des choses» ou la "voix de la vérité». En d'autres termes, CEdipe n'affronte que la vérité, les choses accomplies ou, mieux encore, "ses actes accomplis», c'est-à-dire la vérité que constitue sa propre vie.

\section{Le savoir constitue-t-il le fondement dernier de l'autorité mantique et politique (378-462) ?}

Cette étape commence par un discours d'Edipe qui comprend deux gestes. Le premier est un geste de désespoir et le second un acte ultime de combat envers son opposant. D'abord il accuse Tirésias d'être un serviteur de Créon, avec qui il monte un complot (378-389). Et ensuite, il «se retire» dans le dernier fondement de son autorité et de sa dignité : sa faculté de penser et de se servir du savoir pour le bien de tous. Autrement 
dit, son "charisme». "C'est moi, dit-il, qui ai sauvé la cité, avec ma

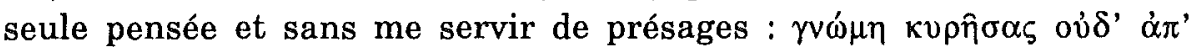

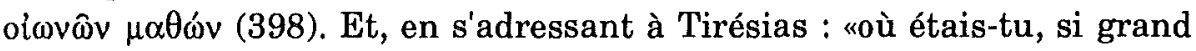
devin, quand la Sphinx était là ? Tu n'as rien dit pour sauver les citoyens ?» (390-396).

Tirésias, impitoyable, après avoir dépassé, sans y répondre, les

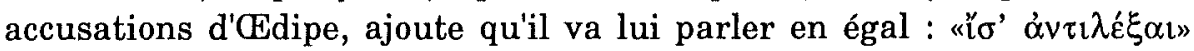
(409) - il est serviteur d'Apollon et il n'a pas besoin d'avoir Créon comme protecteur (410) -, et continue à révéler la vie et les erreurs d'Edipe : «Connais-tu ton origine ? Sais-tu avec qui tu habites ? Tu es ennemi des tiens, morts et vivants. Tu seras égal à tes enfants" (414425). Et après avoir échangé quelques propos très durs qui consacrent l'affaiblissement continu de l'autorité d'CEdipe (428-446), Tirésias lance son dernier discours reprenant, pour la première fois, les événements à venir. Et avant de partir, en s'adressant toujours à Edipe, il ajoute : «Va donc, et pense à cela, et si tu me trouves menteur, tu diras que je ne connais rien en art mantique» (460-462). Le triomphe de Tirésias est clair. En révélant la vérité, en démontrant sa capacité de satisfaire le besoin de la cité en savoir, il restitue à son autorité sa valeur. Il a établi la vérité et par là même rétabli son autorité et son identité blessées par Edipe. L'autorité politique et même charismatique d'CEdipe est presque anéantie. À présent, c'est le cœur qui intervient avec ses doutes et ses peurs. Tirésias a prononcé son verdict.

Il devient donc clair, par cette dernière étape, que pour ces deux hommes de grande autorité, le savoir constitue le dernier niveau et le dernier moyen de lutte et, par conséquent, le fondement dernier aussi bien de l'autorité mantique que politique. Edipe, après avoir perdu un à un les fondements de son autorité - pouvoir d'un chef légitime, autorité d'un sauveur de la cité -, se réfugie dans sa dernière position de combat : sa capacité de se servir du savoir au profit de tous. Si Tirésias se montre plus fort, c'est parce que dans ce cas c'est lui qui sait. Le seul fondement de son autorité a toujours été son savoir divinatoire. Il se présente comme la voix de la vérité, et c'est pour cette raison qu'il peut se placer au même niveau d'autorité qu'un roi tel qu'Edipe. Et s'imposer.

\section{Conclusion}

Si l'autorité, comme nous pouvons le comprendre en lisant Engels et Marx, est présente en tant que rapport de force dans toute forme de rapport social, économique, politique, idéologique et significatif, acception que $\mathrm{P}$. Bourdieu a traitée à la lumière de $\mathrm{M}$. Weber, et si, en partant 
également de M. Weber, nous appréhendons ce même rapport comme "chance" et/ou comme "possibilité", nous constatons, en étudiant l'exemple précédent, que, lorsqu'il s'agit de rapport entre les hommes, l'autorité trouve, de toute manière, implicitement ou explicitement, un fondement dans une forme quelconque de savoir. Certes, cette constatation ne peut être retenue, ici, tant sur le plan de la théorie que sur celui de l'analyse empirique, que comme une hypothèse de travail.

Deux remarques pour conclure. D'un point de vue méthodologique, il serait utile d'étudier les rapports entre une œuvre tragique, le milieu qui l'a vue naître et des réflexions d'ordre théorique. Si l'on envisage la réalité historique de la période classique, il faut souligner que les œuvres tragiques jouent un grand rôle dans le processus de rationalisation et de démocratisation de la vie politique de l'époque. Enfin, remarquons qu'Edipe concrétise le passage de la pensée mythique au logos. C'est par sa seule pensée, sans l'aide de présages, qu'il a vaincu

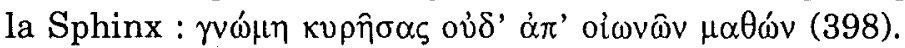

Département de Sciences politiques

Antonios PAPARIZOS

et d'Études internationales

PANTEIOS École des Sciences politiques

Sygrou 136

GR - 17671 ATHÈNES 